\title{
Entanglement and properties *
}

\author{
GianCarlo Ghirardi ${ }^{\dagger}$ \\ Department of Theoretical Physics of the University of Trieste, and \\ Istituto Nazionale di Fisica Nucleare, Sezione di Trieste, Italy and \\ International Centre for Theoretical Physics, Trieste, Italy \\ and \\ Luca Marinatto ${ }^{\ddagger}$ \\ Department of Theoretical Physics of the University of Trieste, and \\ Istituto Nazionale di Fisica Nucleare, Sezione di Trieste, Italy.
}

\begin{abstract}
Various topics concerning the entanglement of composite quantum systems are considered with particular emphasis concerning the strict relations of such a problem with the one of attributing objective properties to the constituents. In particular we will focus our attention to composite quantum systems composed of identical constituents, with the purpose of dealing with subtle issues, which have never been adequately discussed in the literature, originating from the true indistinguishability of the subsystems involved.
\end{abstract}

\section{Introduction}

One of the crucial points of any theory aiming to account for natural phenomena concerns the possibility of identifying the properties objectively possessed by individual physical systems and/or by their constituents. Such a problem acquires a completely different status within different theoretical schemes, typically in the classical and quantum cases. First of all, quantum mechanics, if the completeness assumption is made, requires a radical change of attitude about the problem of attributing objective properties to physical systems due to its fundamentally probabilistic character. Secondly, and even more important for our analysis, it gives rise to specific and puzzling situations concerning the properties of the constituents of a composite system due to its peculiar feature, Entanglement - the direct English translation of the original German form Verschrankung used by Schrödinger [1] - which Schrödinger himself considered "the characteristic trait of Quantum Mechanics, the one that enforces its entire departure from classical line of thoughts". Quantum entanglement has played a central role in the historical development of quantum mechanics, since it has compelled the scientific community to face the essentially nonlocal features of natural processes. Nowadays, entangled states have become the essential ingredients of all processes involving teleportation and quantum cryptography and constitute an important tool for implementing efficient quantum algorithms. This explains why

\footnotetext{
${ }^{*}$ Work supported in part by Istituto Nazionale di Fisica Nucleare, Sezione di Trieste, Italy

${ }^{\dagger}$ e-mail: ghirardi@ts.infn.it

‡e-mail: marinatto@ts.infn.it
} 
a great deal of efforts has been spent by theorists during the last years in trying to characterize the very nature and properties of entanglement, and this is also the reason which motivates our attempt to deepen some questions about these matters.

\section{Properties of individual physical systems}

Let us therefore start by discussing the notion of state of an individual physical system within an hypothetical physical theory. The crucial point, from a conceptual point of view, consists in identifying which is the most accurate characterization that the theory allows concerning the situation of such an individual physical system. For our present purposes we assume that such a "most exhaustive knowledge" is, in principle, possible and that it is expressed by mathematical entities which we will denote as the States of the theory (the so called completeness assumption).

One can immediately exhibit some elementary examples of what we have in mind. For instance, the States of a system of $N$ point particles within Newtonian mechanics are the points $P$ of the $6 N$-dimensional phase space of the system. Within such a theory all conceivable observables, both referring to the whole system as well as to all its subsystems, are functions of the positions and momenta of the particles, so that, when one knows the State of the system, i.e., the phase space point associated to it, one knows also the precise values of all physical observables. Therefore we can claim that within Classical Mechanics all properties are objectively possessed, in the precise sense that the measurement of any given observable simply reveals the pre-existing value possessed by the observable. It goes without saying that if we lack the complete information about the system, then we can make statements only concerning the (epistemic) probabilities that it possesses precise properties. Nevertheless, it remains true that any individual system (and its subsystems) has all conceivable properties, in spite of the fact that we can be ignorant about them ${ }^{1}$.

As everybody knows, the situation is quite different in (non-relativistic) quantum mechanics where one asserts, with the completeness assumption, that the States of a system of $N$ particles are the state vectors $|\psi(1, \ldots, N)\rangle$ of the associated Hilbert space. Accordingly, the theory, in general, consents to make only nonepistemic probabilistic predictions about the outcomes of measurement processes even when the State of the system is known. However, in such a case, there are always complete sets of commuting observables such that the theory attaches probability one to a precise outcome in a measurement process of any one of them. It is then natural to assume (as we will do) that when we can make certain (i.e. with probability one) predictions about the outcomes, the system possesses objectively the property, or element of physical reality "such an observable has such a value", independently of our decision to measure it. Here we have used the expression objective properties and elements of reality with the same meaning that Einstein [2] gave them in the analysis of the EPR paradox:

If, without in any way disturbing a system, we can predict with certainty (i.e. with a probability equal to unity) the value of a physical quantity, then there exists an element of physical reality corresponding to this physical quantity.

\footnotetext{
${ }^{1}$ Stated differently, claims of the kind "the energy of this particle has this specific value" have truth values, i.e. they are definitely either true or false.
} 
When one takes into account the just outlined situation, one can concisely express the lesson that quantum mechanics has taught us, by stating that within such a theory one cannot consider (even in principle) an individual physical system as possessing objectively too many properties. Some of them can be legitimately considered as actual, all the other have the ontological status of potentialities. At any rate, according to the above remarks, a system in a pure state always has complete sets of definite and objective properties.

Up to this point we have confined our attention to quantum systems considered as a whole. However the phenomenon of quantum entanglement makes the situation much more puzzling when consideration is given to composite quantum systems and one raises the problem of the properties of their constituents. As we will see, in such a case it is very common to meet situations (most of which arise as a consequence of the interactions between the constituents) in which the constituents themselves do not possess any property whatsoever. This is a new feature which compels us to face a quite peculiar state of affairs: not only must one limit drastically the actual properties of physical systems (being in any case true that the system as a whole always has some properties), but one is forced also to accept that the parts of a composite system can have no property at all. In this way the quantum picture of the universe as an "unbroken whole", or as "undivided", emerges.

\section{$3 \quad$ Entanglement of two distinguishable particles}

In this section we study the Entanglement between two distinguishable particles $\mathcal{S}_{1}$ and $\mathcal{S}_{2}$. Let us suppose that the two particles are parts of a larger quantum system $\mathcal{S}=\mathcal{S}_{1}+\mathcal{S}_{2}$, whose associated Hilbert space $\mathcal{H}$ is the direct product of the Hilbert spaces of the single subsystems, $\mathcal{H}=\mathcal{H}_{1} \otimes \mathcal{H}_{2}$. We will always assume that the composite quantum system $\mathcal{S}$ is described by a state vector $|\psi(1,2)\rangle \in \mathcal{H}$ or, in a totally equivalent manner, by a pure density operator $\rho=|\psi(1,2)\rangle\langle\psi(1,2)|$.

Let us start by characterizing a non-entangled composite system by making explicit reference to the fact that one of its two constituent subsystems possesses complete sets of properties (as we will see this in turn implies that the same is true for the other constituent):

Definition 3.1 The system $\mathcal{S}_{1}$, subsystem of a composite quantum system $\mathcal{S}=\mathcal{S}_{1}+\mathcal{S}_{2}$ described by the pure density operator $\rho$, is non-entangled with subsystem $\mathcal{S}_{2}$ if there exists a projection operator $P^{(1)}$ onto a one-dimensional manifold of $\mathcal{H}_{1}$ such that:

$$
\operatorname{Tr}^{(1+2)}\left[P^{(1)} \otimes I^{(2)} \rho\right]=1 .
$$

The fact that in the case of non-entangled states it is possible to consider each one of the constituents as possessing complete sets of well definite physical properties, independently of the existence of the other part, follows directly from the following theorem (for the proofs of the present and the following theorems see [3]):

Theorem 3.1 If consideration is given to a composite quantum system $\mathcal{S}=\mathcal{S}_{1}+\mathcal{S}_{2}$ described by the pure state vector $|\psi(1,2)\rangle$ (or, equivalently by the pure density operator $\rho=|\psi(1,2)\rangle\langle\psi(1,2)|$ ) of $\mathcal{H}=\mathcal{H}_{1} \otimes \mathcal{H}_{2}$, each of the following three conditions is necessary and sufficient in order that subsystem $\mathcal{S}_{1}$ is non-entangled with subsystem $\mathcal{S}_{2}$ : 
1. there exists a projection operator $P^{(1)}$ onto a one-dimensional manifold of $\mathcal{H}_{1}$ such that $\operatorname{Tr}^{(1+2)}\left[P^{(1)} \otimes I^{(2)} \rho\right]=1 ;$

2. the reduced statistical operator $\rho^{(1)}=\operatorname{Tr}^{(2)}[\rho]$ of subsystem $S_{1}$ is a projection operator onto a one-dimensional manifold of $\mathcal{H}_{1}$;

3. the state vector $|\psi(1,2)\rangle$ is factorizable, i.e., there exist a state $|\phi(1)\rangle \in \mathcal{H}_{1}$ and a state $|\xi(2)\rangle \in \mathcal{H}_{2}$ such that $|\psi(1,2)\rangle=|\phi(1)\rangle \otimes|\xi(2)\rangle$.

Therefore if a quantum system composed of two subsystems is non-entangled, the states of subsystems $\mathcal{S}_{1}$ and $\mathcal{S}_{2}$ are completely specified, in the sense that it is possible to associate to each of them a unique and well-defined state vector. According to our previous discussion, the individual subsystems can therefore be thought of as having complete sets of definite and objective properties of their own.

We pass now to analyze the case of composite systems of two subsystems in entangled states. According to Theorem 3.1, the reduced density operator of each subsystem is not a projection operator onto a one dimensional manifold. It is then useful to analyze whether there exist projection operators on manifolds of dimension greater than or equal to 2 of $\mathcal{H}_{1}$, satisfying condition (3.1). As shown by the following theorem, there is a strict relation between such projection operators and the range $\mathcal{R}\left[\rho^{(1)}\right]$ of the reduced statistical operator $\rho^{(1)}$ :

Theorem 3.2 A necessary and sufficient condition for the projection operator $P_{\mathcal{M}_{1}}^{(1)}$ onto the linear manifold $\mathcal{M}_{1}$ of $\mathcal{H}_{1}$ to satisfy the two following conditions:

1. $\operatorname{Tr}^{(1)}\left[P_{\mathcal{M}_{1}}^{(1)} \rho^{(1)}\right]=1$;

2. there is no projection operator $\tilde{P}^{(1)}$ of $\mathcal{H}_{1}$ satisfying the conditions $\tilde{P}^{(1)}<P_{\mathcal{M}_{1}}^{(1)}$ (i.e. it projects onto a proper submanifold $\mathcal{N}_{1}$ of $\mathcal{M}_{1}$ ) and $\operatorname{Tr}^{(1)}\left[\tilde{P}^{(1)} \rho^{(1)}\right]=1$,

is that the range $\mathcal{R}\left[\rho^{(1)}\right]$ of the reduced statistical operator $\rho^{(1)}$ coincides with $\mathcal{M}_{1}$.

Let us analyze in detail the consequences of the above theorem by studying the following two cases concerning the range of the reduced statistical operator $\rho^{(1)}$ :

1. $\mathcal{R}\left[\rho^{(1)}\right]=\mathcal{M}_{1} \subset \mathcal{H}_{1}$,

2. $\mathcal{R}\left[\rho^{(1)}\right]=\mathcal{H}_{1}$,

In the first case, given any self-adjoint operator $\Omega^{(1)}$ of $\mathcal{H}_{1}$ which commutes with $P_{\mathcal{M}_{1}}^{(1)}$, if consideration is given to the subset $\mathcal{B}$ (a Borel set) of its spectrum coinciding with the spectrum of its restriction $\Omega_{R}=P_{\mathcal{M}_{1}}^{(1)} \Omega^{(1)} P_{\mathcal{M}_{1}}^{(1)}$ to $\mathcal{M}_{1}$, we can state that subsystem $\mathcal{S}_{1}$ has the objective (in general unsharp) property that $\Omega^{(1)}$ has a value belonging to $\mathcal{B}$. In particular, all operators which have $\mathcal{M}_{1}$ as an eigenmanifold, have a precise objective value. Therefore, even though in the considered case we cannot say that subsystem $\mathcal{S}_{1}$ has a complete set of objective properties, it still has some sharp or unsharp properties associated to any observable which commutes with $P_{\mathcal{M}_{1}}^{(1)}$

On the contrary, in the second case, we have to face the puzzling implications of entanglement in their full generality. In fact the only projection operator $P^{(1)}$ on $\mathcal{H}_{1}$ satisfying $\operatorname{Tr}^{(1)}\left[P^{(1)} \rho^{(1)}\right]=$ 
1 is the identity operator $I^{(1)}$ on the Hilbert space $\mathcal{H}_{1}$. The physical meaning of this fact amounts to state that subsystem $\mathcal{S}_{1}$ does not possess objectively any sharp or unsharp property, i.e., that there is no self-adjoint operator for which one can claim with certainty that the outcome of its measurement will belong to any proper subset of its spectrum.

Another consequence of the entanglement of a composite quantum system is the occurrence of strict correlations between observables of the component subsystems, even when they are far apart and non-interacting. This is expressed by the following theorem:

Theorem 3.3 Subsystem $\mathcal{S}_{1}$ is non-entangled with subsystem $\mathcal{S}_{2}$ iff, given the pure state $|\psi(1,2)\rangle$ of the composite system, the following equation holds for any pair of observables $A(1)$ of $\mathcal{H}_{1}$ and $B(2)$ of $\mathcal{H}_{2}$ such that $|\psi(1,2)\rangle$ belongs to their domains:

$$
\langle\psi(1,2)|A(1) \otimes B(2)| \psi(1,2)\rangle=\left\langle\psi(1,2)\left|A(1) \otimes I^{(2)}\right| \psi(1,2)\right\rangle\left\langle\psi(1,2)\left|I^{(1)} \otimes B(2)\right| \psi(1,2)\right\rangle .
$$

The equation (3.2) implies that no correlation exists between such pairs of observables.

Before passing to analyse the more interesting case of quantum systems composed of identical constituents, we outline that all the previous arguments, definitions and theorems can be easily generalized to systems composed of many distinguishable particles [3].

\section{Entanglement of two identical particles}

The issue of attributing objective properties to the constituents of a quantum system composed of identical particles, unfortunately does not turn out to be a straightforward generalization of the just analysed case involving distinguishable particles, and the problem of entanglement has to be reconsidered.

For example, the naive idea that the two systems being non-entangled requires and is guaranteed by the fact that their state vector is the direct product of vectors belonging to the corresponding Hilbert spaces, cannot be simply transposed to the case of interest. One can easily realize that this must be the case by taking into account that the only allowed states for a system of two identical particles must exhibit precise symmetry properties under the exchange of the two particles. If one would adopt the previous criterion one would be led to conclude (mistakenly) that non-entangled states of identical particles cannot exist. The inappropriateness of taking such a position derives from not taking into account various fundamental facts, in particular that identical particles are truly indistinguishable, so that one cannot pretend that a particular one of them has properties, and that the set of observables for such a system has to be restricted to the self-adjoint operators which are symmetric for the exchange of the variables referring to the two subsystems. Accordingly, it goes without saying that, when dealing with the system, e.g., of two electrons, we will never be interested in questions like "is the electron which we have labeled 1 at a certain position or is its spin aligned with a given axis?" but our only concerns will be of the type: on the basis of the knowledge of the state vector describing the composite system, can one legitimately consider as objective a statement of the kind "there is an electron in a certain region and it has its spin up along a considered direction"?

To prepare the ground for settling such issues, we begin by discussing the case of two identical particles, linking the idea that they are non-entangled to the request that both of them possess a complete set of properties. Accordingly we give the following definition: 
Definition 4.1 The identical constituents $\mathcal{S}_{1}$ and $\mathcal{S}_{2}$ of a composite quantum system $\mathcal{S}=\mathcal{S}_{1}+\mathcal{S}_{2}$ are non-entangled when both constituents possess a complete set of properties.

Taking into account this fact we need to identify the conditions under which one can legitimately claim that one of the constituents possesses a complete set of properties.

Definition 4.2 Given a composite quantum system $\mathcal{S}=\mathcal{S}_{1}+\mathcal{S}_{2}$ of two identical particles described by the pure density operator $\rho$, we will say that one of the constituents has a complete set of properties iff there exists a one dimensional projection operator $P$, defined on the Hilbert space $\mathcal{H}^{(1)}$ of each of the subsystems, such that:

$$
\operatorname{Tr}^{(1+2)}[E(1,2) \rho]=1
$$

where

$$
E(1,2)=P^{(1)} \otimes\left[I^{(2)}-P^{(2)}\right]+\left[I^{(1)}-P^{(1)}\right] \otimes P^{(2)}+P^{(1)} \otimes P^{(2)} .
$$

The operator $E(1,2)$ is symmetric under the exchange of the labels of the two particles and it is a projection operator. Furthermore $\operatorname{Tr}^{(1+2)}[E(1,2) \rho]$ gives the probability of finding at least one of the two identical particles in the state onto which the one-dimensional operator $P$ projects $^{2}$.

We are now able to link the fact that one constituent possesses a complete set of properties to the explicit form of the state vector. This is specified by the following theorem:

Theorem 4.1 One of the identical constituents of a composite quantum system $\mathcal{S}=\mathcal{S}_{1}+\mathcal{S}_{2}$, described by the pure normalized state $|\psi(1,2)\rangle$ has a complete set of properties iff $|\psi(1,2)\rangle$ is obtained by symmetrizing or antisymmetrizing a factorized state.

There follows that the process of symmetrization or antisymmetrization of a factorized quantum state describing a system composed of identical particles does not forbid to attribute a complete set of physical properties to the subsystems: the only claim that one cannot make is to attribute the possessed property to one rather than to the other constituent. At this point it is straightforward to derive the following two theorems which give the desired sufficient and necessary conditions in order that a couple of fermion and boson particles can be considered as non-entangled:

Theorem 4.2 The identical fermions $\mathcal{S}_{1}$ and $\mathcal{S}_{2}$ of a composite quantum system $\mathcal{S}=\mathcal{S}_{1}+\mathcal{S}_{2}$ described by the pure normalized state $|\psi(1,2)\rangle$ are non-entangled iff $|\psi(1,2)\rangle$ is obtained by antisymmetrizing a factorized state.

Theorem 4.3 The identical bosons of a composite quantum system $\mathcal{S}=\mathcal{S}_{1}+\mathcal{S}_{2}$ described by the pure normalized state $|\psi(1,2)\rangle$ are non-entangled iff either the state is obtained by symmetrizing a factorized product of two orthogonal states or if it is the product of the same state for the two particles.

\footnotetext{
${ }^{2}$ We remark that one could drop the last term in the expression (4.2) getting an operator whose expectation value would give the probability of precisely one particle having the properties associated to $P$. In the case of identical fermions this would make no difference but for bosons it would not cover the case of both particles having precisely the same properties.
} 


\section{$5 \quad$ An illuminating example}

Let us consider a system of two identical spin $1 / 2$ particles. We notice that if one would confine his attention to the spin degrees of freedom alone, then, following our definitions and theorems, one would be led to conclude that the singlet state, which can be obtained by antisymmetrizing, e.g., the state $|z \uparrow\rangle_{1}|z \downarrow\rangle_{2}$, would be a non-entangled state. How does this fit with the general (and correct) position that such a state is, in a sense, the paradigmatic case of an entangled two body system? The apparent contradiction can be easily solved by taking into account also the position of the particles, in addition to the spin degrees of freedom. Let us for example consider the state which is obtained by antisymmetrizing the factorized state $|z \uparrow\rangle_{1}|R\rangle_{1}|z \downarrow\rangle_{2}|L\rangle_{2}$ :

$$
|\psi(1,2)\rangle=\frac{1}{\sqrt{2}}\left[|z \uparrow\rangle_{1}|R\rangle_{1}|z \downarrow\rangle_{2}|L\rangle_{2}-|z \downarrow\rangle_{1}|L\rangle_{1}|z \uparrow\rangle_{2}|R\rangle_{2}\right]
$$

where $|R\rangle$ and $|L\rangle$ are two orthogonal spatial locations. Even though for such a state it is meaningless to speak of particle 1 as distinguishable from particle 2 , we can correctly state that there is a particle with spin up along $z$-axis and located in region $R$ and that there is a particle with spin down and located in region L. Having attributed a complete set, i.e. position and spin, of properties to both constituents, we can correctly state that the above state in non-entangled, notwithstanding its non-factorized mathematical form.

On the contrary, for a state like

$$
|\psi(1,2)\rangle=\frac{1}{\sqrt{2}}\left[|z \uparrow\rangle_{1}|z \downarrow\rangle_{2}-|z \downarrow\rangle_{1}|z \uparrow\rangle_{2}\right] \otimes\left[|R\rangle_{1}|L\rangle_{2}+|L\rangle_{1}|R\rangle_{2}\right],
$$

which cannot be obtained by antisymmetrizing a factorized state, it is not possible, for example, to attribute any definite spin property to the particle located in $R$ and equivalently no definite spatial property can be attributed to the particle with spin up. In the case where $|R\rangle$ and $|L\rangle$ correspond to two distant spatial locations, the state vector of Eq. (5.2) represents the paradigmatic state considered in the usual EPR argument and in the experiments devised to reveal the non-local features of quantum mechanics.

The picture should now be clear: no state of two fermions in the singlet spin state can be obtained by antisymmetrizing a factorized wave function, when also the remaining degrees of freedom are taken into account. In this sense one can understand how there is no contradiction between the usual statement that the singlet state is entangled and the fact that, if one disregards the spatial degrees of freedom, it can be obtained by antisymmetrizing a factorized spin state.

\section{Entanglement of $N$ indistinguishable particles}

Let us pass now to the case of $N$ indistinguishable particles and let us try to define, in a conceptually correct way, the idea that the set of $N$ identical particles we are dealing with, can be partitioned into two "subsets" of cardinality $M$ and $K$, which are non-entangled with each other. By following strictly the procedure we have introduced for the case of two particles, we will do this by first considering the possibility of attributing a complete set of properties to each subset and we will give the following definition: 
Definition 6.1 Given a quantum system of $N$ identical particles described by a pure state $\left|\psi^{(N)}\right\rangle$ we will say that it contains two non-entangled "subgroups" of particles of cardinality $M$ and $K,(M+K=N)$, when both subgroups possess a complete set of properties.

In order to make statements of the sort we are interested in, i.e., that objective properties can be attached to the subsets associated to a partition of the particles or, equivalently, that such subsets are non-entangled among themselves, we have to impose quite strict constraints on the state vectors associated so such subsets (confining for brevity our attention to the case of fermion systems only ${ }^{3}$ ). To this purpose we will begin by saying that the states $\left|\Phi^{(K)}\right\rangle$ and $\left|\Sigma^{(M)}\right\rangle$ belonging to $\mathcal{H}_{A}^{(K)}$ and $\mathcal{H}_{A}^{(M)}$ (the antisymmetric manifolds of the Hilbert spaces of $K$ and $M$ particles respectively) are one-particle orthogonal iff the following condition holds

$$
\int d X \Sigma^{(M)}(1, \ldots, X, \ldots, M) \Phi^{(K) \star}(1, \ldots, X, \ldots, K)=0 \quad \forall X
$$

for every possible choice of the unsaturated variables.

It is possible to prove that the above condition is equivalent to assuming the existence of a single particle basis $\left\{\left|\phi_{i}\right\rangle\right\}$ such that the Fourier expansion of $\left|\Phi^{(K)}\right\rangle$ involves only single particle states whose indices belong to $\Delta$, and $\left|\Sigma^{(M)}\right\rangle$ involves only states whose indices belong to $\Delta^{\star}$, where $\Delta$ and $\Delta^{\star}$ are two disjoint partition of the set of indices $i$. For the sake of brevity we omit to describe the rather cumbersome procedure for identifying the generalization of the projection operator of Eq. (4.2), which allows us to speak of properties objectively possessed by the considered subgroups of particles, and we pass directly to stating the final interesting result:

Theorem 6.1 Given a system $S^{(N)}$ of $N$ identical fermions in a pure state $\left|\psi^{(N)}\right\rangle$ of $\mathcal{H}_{A}^{(N)}$ it contains two non-entangled subsets of cardinality $M$ and $K$ iff $\left|\psi^{(N)}\right\rangle$ can be written as:

$$
\left|\psi^{(N)}\right\rangle=\sqrt{\left(\begin{array}{l}
N \\
K
\end{array}\right)} P_{A}\left[\left|\Pi^{(M)}(1, \ldots, M)\right\rangle \otimes\left|\Phi^{(K)}(M+1, \ldots, N)\right\rangle\right],
$$

where the states $\left|\Pi^{(M)}(1, \ldots, M)\right\rangle$ and $\left|\Phi^{(K)}(M+1, \ldots, N)\right\rangle$ are "one-particle orthogonal" among themselves and $P_{A}$ is the projection operator onto the totally antisymmetric manifold $\mathcal{H}_{A}^{(N)}$.

This theorem represents the correct generalization of the one already proved for the simpler case of two identical fermions, and once again it makes precise the mathematical conditions under which an arbitrary $N$-particle state can (or cannot) describe entangled subgroups of particles. It is worth noticing that, once more, the non-factorized form of the state required by the antisymmetrization postulate, does not imply by itself the entanglement of the subsystems.

\section{One-particle orthogonality}

Let us now discuss the physical motivations for the request of the one-particle orthogonality in dealing with non-entangled subgroups of identical particles. To this purpose, let us consider two closed submanifolds $V_{\Delta}^{(M)}$ and $V_{\Delta^{*}}^{(K)}$ of $\mathcal{H}_{A}^{(M)}$ and $\mathcal{H}_{A}^{(K)}$ respectively, spanned by one-particle

\footnotetext{
${ }^{3}$ For a more general and detailed analysis of all the mathematical intricacies, which also includes the boson case, we address the interested reader to the original paper [3].
} 
orthogonal states, as defined previously. If $\left\{\left|\Upsilon_{j}^{(M)}\right\rangle\right\}$ and $\left\{\left|\Xi_{l}^{(K)}\right\rangle\right\}$ are two orthonormal bases spanning such manifolds, given arbitrary states $\left|\chi^{(M)}\right\rangle$ and $\left|\tau^{(M)}\right\rangle$ of $V_{\Delta}^{(M)},\left|\mu^{(K)}\right\rangle$ and $\left|\nu^{(K)}\right\rangle$ of $V_{\Delta^{*}}^{(K)}$, the following relations hold:

$$
\sum_{l}\left|\left[\sqrt { ( \begin{array} { l } 
{ N } \\
{ K }
\end{array} ) } \left\langle\left.\chi^{(M)}\left|\left\langle\Xi_{l}^{(K)}\right| P_{A}\right] \cdot\left[\sqrt{\left(\begin{array}{l}
N \\
K
\end{array}\right)} P_{A}\left|\tau^{(M)}\right\rangle\left|\nu^{(K)}\right\rangle\right]\right|^{2}=\left|\left\langle\chi^{(M)} \mid \tau^{(M)}\right\rangle\right|^{2},\right.\right.\right.
$$

and

$$
\sum_{j}\left|\left[\sqrt { ( \begin{array} { l } 
{ N } \\
{ K }
\end{array} ) } \left\langle\left.\Upsilon_{j}^{(M)}\left|\left\langle\mu^{(K)}\right| P_{A}\right] \cdot\left[\sqrt{\left(\begin{array}{l}
N \\
K
\end{array}\right)} P_{A}\left|\tau^{(M)}\right\rangle\left|\nu^{(K)}\right\rangle\right]\right|^{2}=\left|\left\langle\mu^{(K)} \mid \nu^{(K)}\right\rangle\right|^{2}\right.\right.\right.
$$

These equations show that, provided two one-particle orthogonal manifolds $V_{\Delta}^{(M)}$ and $V_{\Delta^{*}}^{(K)}$ can be identified, and provided the interactions between the particles determining the subsequent evolution do not alter the specific features of the state vector, then one can do the physics within each manifold by disregarding the other one, even though the appropriate antisymmetrization requests for the whole set of fermions are respected ${ }^{4}$. In fact scalar products between antisymmetrized products of one-particle orthogonal states turns out to be equivalent to the evaluation of reduced scalar products involving only states of a certain subgroup of all the particles, neglecting the presence of the others.

These considerations should have made clear the appropriateness of adopting our criteria for the attribution of complete sets of properties associated to $\left|\Pi^{(M)}\right\rangle$ and $\left|\Phi^{(K)}\right\rangle$ and for the identification of non-entangled subsets of a system of $N$ identical fermions.

\section{Some remarks concerning almost perfect non-entanglement}

Having made precise the idea of a "group of particles" of a system of identical particles being non-entangled with the remaining ones, we can analyse the following physical situation: there is a Helium atom at the origin $O$ of our reference frame (here) and a Lithium atom at a distance $d$ from $O$ (there). Our worries concern the legitimacy of claiming "there is a Helium atom at the origin" or "there is a Lithium atom at a distance $d$ from the origin" when one takes into account the identity of the electrons of the two systems which requires the state vector to be totally antisymmetric under their exchange ${ }^{5}$. In order to settle the issue let us pay attention to the total state vector of the complete system "Helium+Lithium":

$$
\left.\left.\left|\psi^{(5)}\right\rangle \propto \mathcal{G}\left[\mid \text { Helium }^{(2)} \text { here }\right\rangle \otimes \mid \text { Lithium }^{(3)} \text { there }\right\rangle\right],
$$

where $\mathcal{G}$ is the permutation operator which exchange at least one of the two particle indices of the Helium with those of the Lithium.

\footnotetext{
${ }^{4}$ With reference to eq.(7.1), we stress that the one-particle orthogonality of the states $\left|\tau^{(M)}\right\rangle$ and $\left|\nu^{(K)}\right\rangle$, and $\left|\chi^{(M)}\right\rangle$ and $\left|\Xi^{(K)}\right\rangle$ as well as the corresponding ones for the states appearing in eq. (7.2), is absolutely fundamental - as the reader can check - in order that the (physically important) equality sign between the expressions at the left and right hand sides of the equations holds.

${ }^{5}$ To discuss the conceptually relevant aspects of this problem we will confine, for simplicity, our considerations only to the electrons which are involved, disregarding the nuclei of the atoms - and the necessary antisymmetrization concerning the protons and the neutrons.
} 
Since the factors $\mid$ Helium $^{(2)}$ here $\rangle$ and $\mid$ Lithium $^{(3)}$ there $\rangle$ do not exactly satisfy our fundamental request of being one-particle orthogonal, due to the non-compactness of their spatial supports, the above state cannot be strictly considered as non-entangled. However we can explicitly evaluate integrals like the one of Eq. (6.1), which, when they vanish, make legitimate precise claims concerning the objective properties possessed by the two subgroups of two and three electrons. We notice that the modulus of such integrals is of the order of the overlap integrals of the electronic wave functions. Since they decrease exponentially outside a region of $10^{-8} \mathrm{~cm}$ from the corresponding nuclei, we immediately see that, for a distance between the two nuclei of the order of $1 \mathrm{~cm}$, the relevant integral turns out to have a value of the order of $10^{-10^{16}}$. So, strictly speaking, our claim "there is a Helium atom around the origin" is not perfectly correct. However, in the considered case, the formal conditions which are necessary for attributing consistently objective properties to "a group of particles" are satisfied to such an high degree of accuracy that we can still legitimately say that "there is a Helium atom here and a Lithium atom there" and that the whole state is (almost) non-entangled.

\section{Conclusions}

We have discussed entanglement from the physical point of view of its implications concerning the properties objectively possessed by subgroups of constituents of a composed physical systems. In the delicate case of physical systems involving identical particles we have given consistent definitions and proved general theorems. They should have clarified that the apparent and formal "entanglement" arising from the (anti)symmetrization of the corresponding wave function does not imply by itself, both from a conceptual and a practical point of view, an actual form of entanglement.

\section{References}

[1] E.Schrödinger, Naturwissenschaften, 23, 807 (1935); English translation in: Proc. Am. Philos. Soc., 124, 323 (1980).

[2] A.Einstein, B.Podolsky and N.Rosen, Phys. Rev., 47, 777 (1935).

[3] G.C.Ghirardi, L.Marinatto and T.Weber, Journal of Statistical Physics, 108, 49 (2002). 